\title{
Efeitos de tamanho de vaso e sistemas de condução no desenvolvimento e qualidade de cultivares de zinia ${ }^{1}$
}

\author{
ANA CHRISTINA ROSSINI PINTO²; TERESINHA DE JESUS DELÉO RODRIGUES ${ }^{3}$; \\ IZABEL CRISTINA LEITE ${ }^{3}$ e JOSÉ CARLOS BARBOSA ${ }^{4}$
}

\begin{abstract}
RESUMO
A zínia tem potencial para ser usada como planta envasada no Brasil, pois apresenta capítulos atrativos, resistência à seca, fácil propagação, rápido crescimento, mínima exigência de tratos culturais e rusticidade. Avaliou-se o efeito do tamanho de vaso $(0,6 \mathrm{~L}$ e $1,3 \mathrm{~L})$ e do sistema de condução (livre brotação de gemas e desponte da gema floral apical) no desenvolvimento e qualidade de cultivares de zínia ('Profusion Cherry', 'Thumbelina', 'Lilliput' e 'Persian Carpet'). As plantas foram cultivadas no outono, em casa de vegetação, em Jaboticabal (SP). O delineamento experimental foi em blocos casualizados com quatro repetições (seis vasos por parcela), e analisado em esquema fatorial $4 \times 2 \times 2$ (quatro cultivares, dois tamanhos de vaso e dois sistemas de condução). A semeadura foi realizada em bandejas de poliestireno e, as mudas, transplantadas no estádio de $2 .^{\circ}$ par de folhas nodais verdadeiras para vasos preenchidos com substrato comercial e fertilizado com Osmocote ${ }^{\circledR}$. Quando a gema floral apical apresentava 3-5 mm, foi retirada manualmente. 'Profusion Cherry' apresentou o melhor desempenho, com plantas compactas e baixas $(18,0 \mathrm{~cm})$, com maior diâmetro de capítulos $(4,74 \mathrm{~cm})$, maior porcentagem de cobertura do vaso pela parte aérea $(87,3 \%)$, maior índice de colheita de folhagem e capítulos $(0,65)$ e, menor ciclo de produção (52 dias). 'Thumbelina' mostrou potencial para uso em vaso, mas apresentou-se inferior a 'Profusion Cherry' em relação à altura das plantas $(23,0 \mathrm{~cm})$, cobertura do vaso $(80,8 \%)$ e duração do ciclo de produção (59
\end{abstract}

dias). 'Lilliput' e 'Persian Carpet' não revelaram aspecto compacto e porte baixo, com altura de plantas de 57,0 e $50,0 \mathrm{~cm}$ respectivamente. Para todos os cultivares, o vaso de $0,6 \mathrm{~L}$ foi mais adequado, considerando-se o desenvolvimento e a qualidade das plantas. O desponte da gema floral apical atrasou o ciclo de produção e não contribuiu para incrementar a qualidade das plantas.

Palavras-chave: Zinnia elegans; Zinnia haageana; crescimento, planta envasada, pós-produção.

\section{ABSTRACT \\ Effect of pot size and growing system on development and quality of potted zinnia cultivars}

Zinnia has good prospect to be used as potted plant in Brazil.It shows attractive flowers, drought resistance, easy propagation, fast growth, minimal labor requirements and rusticity. The present study evaluated the effects of pot size $(0.6$ and $1.3 \mathrm{~L})$ and growing system (free sprout of buds and apical flower bud pinch) on zinnia cultivars ('Profusion Cherry', 'Thumbelina', 'Lilliput' and 'Persian Carpet') development and quality. Plants were grown in the fall, in greenhouse, at Jaboticabal, State of São Paulo, Brazil. The experimental design was a randomized blocks, with four replicates (six pots per experimental unit), in factorial arrangement $4 \times 2 \times 2$ (four cultivars, two pot sizes and two growing systems). Seeds

\footnotetext{
${ }^{1}$ Extraído da tese de doutorado da primeira autora, UNESP, Jaboticabal (SP).

${ }^{2}$ Bolsista do CNPq. Email: achrisp@ig.com.br

${ }^{3}$ Departamento de Biologia Aplicada à Agropecuária, UNESP, 14884-900 Jaboticabal (SP).

${ }^{4}$ Departamento de Ciências Exatas, UNESP, 14884-900 Jaboticabal (SP).
} 
were sown in polystyrene plug trays. Seedlings were transplanted, at second nodal true leaves stage to pots filled with commercial media and fertilized with Osmocote. When apical flower bud had $3-5 \mathrm{~mm}$ it was manualy removed. 'Profusion Cherry' showed the best performance among the cultivars with a compact and short aspect $(18.0 \mathrm{~cm})$, larger flower diameter $(4.74 \mathrm{~cm})$, larger pot coverage by canopy $(87.3 \%)$, larger foliage and flowers harvest index (0.65) and, a shorter production cycle (52 days). 'Thumbelina' showed potential as potted plant, but was inferior to 'Profusion Cherry' regarding to plant height $(23.0 \mathrm{~cm})$, pot coverage $(80.8 \%)$ and, production cycle (59 days). 'Lilliput' and 'Persian Carpet' did not show a compact and short aspect, with plants height of 57.0 and $50.0 \mathrm{~cm}$, respectively. For all cultivars, the $0.6 \mathrm{~L}$ pot was more appropriated, considering plant development and quality. Apical flower bud pinch delayed production cycle and did not improve plants quality.

Key-words: Zinnia elegans; Zinnia haageana; growth, potted plant, postproduction.

\section{INTRODUÇÃO}

As zínias são plantas anuais floríferas utilizadas em jardins residenciais e públicos, no mundo todo, e cultivadas para a produção de flores de corte (BOYLE et al., 1986). Embora não sejam comumente comercializadas envasadas no Brasil, como ocorre em alguns países do hemisfério Norte, apresentam características interessantes à utilização em vaso, como alto valor ornamental em virtude da ampla variedade de cores, tamanho e forma dos capítulos, resistência à seca, produção significativa de capítulos por planta, rápido desenvolvimento, fácil cultivo, necessidade de tratos culturais mínimos e rusticidade (PINTO, 2003). Ademais, a utilização de espécies rústicas permite o uso de técnicas de cultivo comuns e menos dispendiosas, possibilitando a redução nos custos de produção, sem perda de qualidade, e disponibilizando, no mercado de flores, um produto acessível a um maior número de pessoas.

Freqüentemente, flores não usuais, ou aquelas apresentadas em uma forma nova, despertam a curiosidade, estimulando o consumo (OPPENHEIM, 2000) e preenchem a necessidade cada vez mais intensa de novidades, visto que mudanças na preferência do consumidor são comuns (BOYLE, 1991). Assim, o estudo da produção de plantas ornamentais cultivadas para fins outros, que não os usuais, é de grande va- lor, contribuindo para que novos produtos sejam lançados no mercado, desde que sejam competitivos e satisfaçam às necessidades dos consumidores.

Geralmente, nas plantas floridas envasadas, busca-se porte baixo, compacto e com boa ramificação, características essas que variam de acordo com diversos fatores, como cultivar, tamanho do vaso e sistema de condução. A utilização e o manejo adequado desses fatores podem contribuir para a obtenção de um produto de qualidade, num período menor de produção, permitindo ao produtor um rápido retorno do capital investido e maior otimização no uso da estrutura de cultivo.

Considerando-se a ausência de informações no Brasil relativas ao desenvolvimento de zínia e à qualidade da planta e flores formadas, quando produzidas em vaso, e a necessidade para o estabelecimento da produção, o presente estudo teve por objetivo verificar os efeitos do tamanho de vaso e sistemas de condução, no desenvolvimento e na qualidade de cultivares de zínia, visando à viabilização de um novo produto para o mercado de flores envasadas.

\section{MATERIAL E MÉTODOS}

O experimento foi conduzido no outono, em casa de vegetação, na Faculdade de Ciências Agrárias e Veterinárias, UNESP, no município de Jaboticabal, SP. Durante o período de produção, desde a semeadura até a floração, as plantas foram mantidas sob fotoperíodo natural (11h56 min a $10 \mathrm{~h} 58 \mathrm{~min}$ ) e temperatura mínima, máxima e média de 6,$0 ; 37,5$ e $23,8 \pm 1^{\circ} \mathrm{C}$ respectivamente. A umidade relativa do ar apresentou variações médias diárias de 28 a 99\%, e a média de radiação fotossinteticamente ativa máxima foi de $565,1 \mu \mathrm{mol} \cdot \mathrm{m}^{-2} \cdot \mathrm{s}^{-1}$.

Mudas de Zinnia elegans Jacq. 'Thumbelina Mix' e 'Lilliput Mix', de Zinnia haageana Regel 'Persian Carpet' e de 'Profusion Cherry' (híbrido de Z. elegans e Z. angustifolia HBK.) foram produzidas por meio de semeadura realizada em bandejas de poliestireno expandido, modelo CM 128-62, colocando-se uma semente por célula. As bandejas foram preenchidas com substrato comercial composto à base de casca de pínus, vermiculita, turfa e matéria orgânica $\left(\mathrm{pH}_{\text {agua }}=6,11 ; \mathrm{N}=0,42 \% ; \mathrm{P}=0,098 \%\right.$; S $=0,22 \% ; \mathrm{K}=1,85 \mathrm{~g} \cdot \mathrm{kg}^{-1} ; \mathrm{Ca}=8,85 \mathrm{~g} \cdot \mathrm{kg}^{-1} ; \mathrm{Mg}=0,9$ g.kg-1 $; \mathrm{Cu}=38,0 \mathrm{mg} \cdot \mathrm{kg}^{-1} ; \mathrm{Fe}=20,54 \mathrm{mg} \cdot \mathrm{kg}^{-1} ; \mathrm{Mn}=$ $285,0 \mathrm{mg} \cdot \mathrm{kg}^{-1} ; \mathrm{Zn}=72,0 \mathrm{mg} \cdot \mathrm{kg}^{-1}$ e condutividade elétrica de $0,84 \mathrm{mmhos} / \mathrm{cm}$, em suspensão de 1,0 parte de substrato para 1,5 parte de água destilada). 
Quando as mudas atingiram o estádio de $2 .^{\circ}$ par de folhas verdadeiras totalmente expandidas, foram transplantadas para vasos, preenchidos com substrato comercial composto por uma mistura de casca de pínus compostado, casca de eucalipto compostado, pó de xaxim compostado, pó de coco lavado, turfa, carvão vegetal moído, casca de arroz esterilizada, vermiculita expandida e argila $\left(\mathrm{pH}_{\text {água }}=6,12 ; \mathrm{N}=\right.$ $0,32 \% ; \mathrm{P}=0,10 \% ; \mathrm{S}=0,24 \% ; \mathrm{K}=1,70 \mathrm{~g} \cdot \mathrm{kg}^{-1} ; \mathrm{Ca}$ $=4,55 \mathrm{~g} \cdot \mathrm{kg}^{-1} ; \mathrm{Mg}=8,2 \mathrm{~g} \cdot \mathrm{kg}^{-1} ; \mathrm{Cu}=37,0 \mathrm{mg} \cdot \mathrm{kg}^{-1} ; \mathrm{Fe}$ $=18,9 \mathrm{mg} \cdot \mathrm{kg}^{-1} ; \mathrm{Mn}=136,0 \mathrm{mg} \cdot \mathrm{kg}^{-1} ; \mathrm{Zn}=75,0 \mathrm{mg} \cdot \mathrm{kg}^{-}$ ${ }^{1}$ e condutividade elétrica de $\left.0,75 \mathrm{mmhos} / \mathrm{cm}\right)$. Adicionou-se ao substrato do vaso, antes do transplante da muda, 1,08 e 1,98 gramas de Osmocote Sierra 1510-10 + micronutrientes respectivamente, no vaso de $0,6 \mathrm{~L}$ e de 1,3 L. No plantio, colocou-se uma muda por vaso, no seu centro.

Efetuou-se o desponte da gema floral apical do caule principal, manualmente, quando ela apresentava 3-5 mm de comprimento. No sistema de condução em livre brotação de gemas, não se retirou nenhuma gema da planta. Utilizaram-se vasos de plástico preto, com capacidade de $0,60 \mathrm{~L}(9,4 \mathrm{~cm}$ de profundidade, diâmetro da base de $9,3 \mathrm{~cm}$ e diâmetro superior de $13,0 \mathrm{~cm})$ e de $1,30 \mathrm{~L}(11,5 \mathrm{~cm}$ de profundidade, diâmetro da base de $11,1 \mathrm{~cm}$ e diâmetro superior de $14,7 \mathrm{~cm}$ ).

As plantas foram irrigadas manualmente, no início da manhã e ao anoitecer, cada vaso recebendo quantidade de água suficiente para manter o substrato úmido.

No estádio de floração, quando as plantas dos cultivares apresentavam de seis a sete capítulos abertos por planta, de cada parcela amostraram-se dois vasos, para determinar a altura do caule principal (medida da superfície do substrato à base do receptáculo floral, em plantas conduzidas em livre brotação, e da superfície do substrato à inserção do nó, do qual se removeu a gema floral apical, em plantas submetidas ao desponte), bem como comprimento dos ramos laterais e a área foliar total.

Estimou-se a área foliar total tomando-se uma amostra de 20 folhas/parcela, as quais foram medidas pelo sistema de análise de imagem digital, Delta T Devices. Com o valor da massa seca das lâminas medidas, determinou-se o valor da área foliar total, utilizando-se o valor da massa seca total de folhas da planta.

Determinou-se a massa seca (g) de folhas, caule principal, ramos laterais, capítulos, botões florais e raízes, as quais foram secas em estufa a $75^{\circ} \mathrm{C}$, até massa constante e as pesagens, realizadas em balança analítica. A massa seca total da planta (g) foi obtida pela soma da massa seca das partes da planta. Determinou-se, então, a distribuição da massa seca entre as partes da planta (\%). O índice de colheita de folhagem e capítulos foi obtido pela relação entre a massa seca de folhas e capítulos e a massa seca total da planta (DAIE, 1985).

O ciclo de produção foi estabelecido, considerando-se o número de dias, desde a semeadura, para que seis a sete capítulos estivessem abertos. Considerou-se como capítulo aberto aquele com as flores liguladas totalmente expandidas e as flores tubuladas abertas.

Avaliou-se o diâmetro dos capítulos, a altura da parte aérea (medida da superfície do substrato ao ponto mais alto da parte aérea) e a largura da parte aérea (medida como a maior largura alcançada, de uma extremidade a outra da parte aérea). A cobertura do vaso pela parte aérea foi avaliada visualmente, com auxílio de um círculo dividido em oito partes, que indicaram a porcentagem de cobertura do vaso pela parte aérea, ao observá-la de cima.

Efetuou-se o delineamento experimental em blocos casualizados com 16 tratamentos e quatro repetições (seis vasos em cada parcela), e analisou-se em esquema fatorial $4 \times 2 \times 2$ (quatro cultivares, dois tamanhos de vaso e dois sistemas de condução). Os dados obtidos foram submetidos à análise da variância (teste F) e as médias de tratamentos, comparadas pelo teste de Tukey ao nível de 5\% de probabilidade.

\section{RESULTADOS E DISCUSSÃO}

Altura e largura da parte aérea, altura do caule principal, comprimento de ramos laterais e área foliar total 'Profusion Cherry' e 'Thumbelina' apresentaram altura da parte aérea e comprimento de ramos laterais significativamente menores em relação aos demais cultivares (Tabela 1), produzindo plantas mais compactas e de aspecto visual superior (Figura 1). Embora o desponte da gema floral apical, no caule principal, tenha proporcionado, para todos os cultivares, menor altura do caule principal (Tabela 2), já que impediu o desenvolvimento da haste floral, os ramos laterais alongaram-se suficientemente para produzir plantas com altura da parte aérea que não diferiu de plantas conduzidas com livre brotação de gemas (Tabela 1). 
Tabela 1. Altura e largura da parte aérea, comprimento de ramos laterais e área foliar total, de cultivares de zínia na floração, desenvolvidos em vasos de diferentes tamanhos e sistemas de condução

\begin{tabular}{|c|c|c|c|c|c|}
\hline \multirow{2}{*}{ Fator } & \multirow{2}{*}{ Variável } & \multicolumn{2}{|c|}{ Parte aérea } & \multirow{2}{*}{$\begin{array}{l}\text { Comprimento de } \\
\text { ramos laterais }\end{array}$} & \multirow{2}{*}{$\begin{array}{l}\text { Área foliar } \\
\text { total }\end{array}$} \\
\hline & & Altura & Largura & & \\
\hline & & & $-\mathrm{cm}$ & & $\mathrm{dm}^{2}$ \\
\hline \multirow[t]{5}{*}{ Cultivar } & 'Profusion Cherry' & $17,98 \mathrm{c}$ & $27,41 \mathrm{~b}$ & $13,77 \mathrm{~b}$ & $4,74 \mathrm{~b}$ \\
\hline & 'Thumbelina' & $22,83 \mathrm{c}$ & $22,22 \mathrm{c}$ & $13,60 \mathrm{~b}$ & $9,93 \mathrm{a}$ \\
\hline & 'Lilliput' & 56,79 a & $28,41 \mathrm{~b}$ & $31,14 \mathrm{a}$ & 9,83 a \\
\hline & 'Persian Carpet' & $49,73 \mathrm{~b}$ & 44,19 a & $32,05 \mathrm{a}$ & $5,67 \mathrm{~b}$ \\
\hline & d.m.s. & 5,22 & 2,57 & 3,16 & 1,43 \\
\hline \multirow[t]{3}{*}{ Tamanho de vaso } & $0,60 \mathrm{~L}$ & $35,85 \mathrm{a}$ & $27,73 \mathrm{~b}$ & $21,76 \mathrm{~b}$ & $6,40 \mathrm{~b}$ \\
\hline & $1,30 \mathrm{~L}$ & $37,82 \mathrm{a}$ & 33,38 a & $23,52 \mathrm{a}$ & 8,69 a \\
\hline & d.m.s. & 2,79 & 1,37 & 1,69 & 0,76 \\
\hline \multirow[t]{3}{*}{ Sistema de condução } & Livre brotação de gemas & $36,23 \mathrm{a}$ & $30,27 \mathrm{a}$ & $22,56 \mathrm{a}$ & $7,21 \mathrm{a}$ \\
\hline & Desponte da gema floral apical & 37,44 a & 30,84 a & $22,72 \mathrm{a}$ & 7,88 a \\
\hline & d.m.s. & 2,79 & 1,37 & 1,69 & 0,76 \\
\hline \multicolumn{2}{|l|}{ Teste F (Cultivar - C) } & $194,71^{* *}$ & $193,41^{* *}$ & $152,86^{* *}$ & $52,14^{* *}$ \\
\hline \multicolumn{2}{|c|}{ Teste F (Tamanho de vaso - V) } & $2,01 \mathrm{~ns}$ & $68,79 * *$ & $4,40^{*}$ & $36,90 * *$ \\
\hline \multicolumn{2}{|c|}{ Teste F (Sistema de condução $-\mathrm{S}$ ) } & $0,77 \mathrm{~ns}$ & $0,72 \mathrm{~ns}$ & $0,04 \mathrm{~ns}$ & $3,11 \mathrm{~ns}$ \\
\hline \multicolumn{2}{|c|}{ Teste F (Interação C x V) } & $1,74 \mathrm{~ns}$ & $6,50 * *$ & $1,40 \mathrm{~ns}$ & $2,03 \mathrm{~ns}$ \\
\hline \multicolumn{2}{|c|}{ Teste F (Interação C x S) } & $0,61 \mathrm{~ns}$ & $1,47 \mathrm{~ns}$ & $0,56 \mathrm{~ns}$ & $1,33 \mathrm{~ns}$ \\
\hline \multicolumn{2}{|c|}{ Teste F (Interação V x S) } & $0,71 \mathrm{~ns}$ & $0,10 \mathrm{~ns}$ & $0,33 \mathrm{~ns}$ & $3,29 \mathrm{~ns}$ \\
\hline \multicolumn{2}{|c|}{ Teste F (Interação C x V x S) } & $0,41 \mathrm{~ns}$ & $1,69 \mathrm{~ns}$ & $0,17 \mathrm{~ns}$ & $1,65 \mathrm{~ns}$ \\
\hline \multicolumn{2}{|c|}{ Coeficiente de variação (\%) } & 15,01 & 8,93 & 14,79 & 20,03 \\
\hline
\end{tabular}

Médias seguidas de letras iguais nas colunas não diferem entre si (Tukey 5\%); ns não significativo a $5 \%$ de probabilidade; * e ${ }^{* *}$ respectivamente, significativo a $5 \%$ e $1 \%$ de probabilidade; d.m.s. = diferença mínima significativa.

Tabela 2. Altura do caule principal, de cultivares de zínia na floração, desenvolvidos em diferentes sistemas de condução

Sistema de condução

Cultivar

Livre brotação

de gemas

Desponte da gema floral apical

d.m.s.

Altura do caule principal

\begin{tabular}{|c|c|c|c|}
\hline \multirow[b]{2}{*}{ 'Profusion Cherry' } & \multicolumn{2}{|c|}{$-\mathrm{cm}-$} & \\
\hline & $12,22 \mathrm{aC}$ & $6,51 \mathrm{bC}$ & \\
\hline 'Thumbelina' & $15,50 \mathrm{aC}$ & $11,43 \mathrm{bB}$ & 2,98 (S d. C) \\
\hline 'Lilliput' & 38,38 aA & $22,87 \mathrm{bA}$ & 3,94 (C d. S) \\
\hline 'Persian Carpet' & $33,23 \mathrm{aB}$ & $22,88 \mathrm{bA}$ & \\
\hline Teste F (Inte1 & condução) & & $12,09^{* *}$ \\
\hline
\end{tabular}


'Profusion Cherry' e 'Persian Carpet' apresentaram menor área foliar total comparados a 'Thumbelina' e 'Lilliput'. Embora 'Profusion Cherry' tenha apresentado menor área foliar comparado ao 'Lilliput', seu aspecto apresentou-se mais compacto em razão do menor comprimento
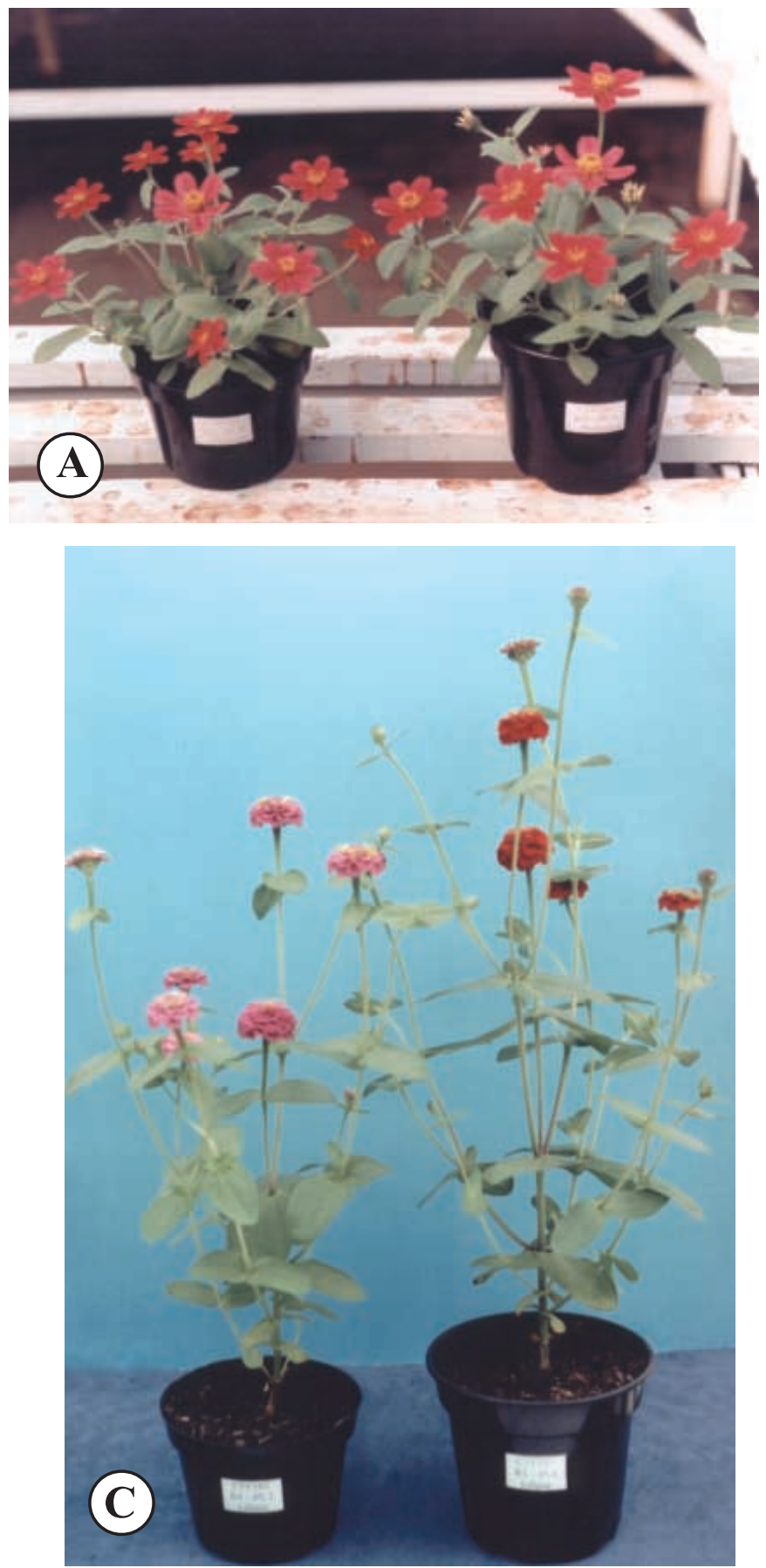

dos ramos laterais (Tabela 1) e da menor altura do caule principal nos dois sistemas de condução (Tabela 2). 'Lilliput' mesmo apresentando maior área foliar total, apresentou os ramos laterais muito longos, produzindo plantas pouco compactas (Tabela 1 e Figura 1).
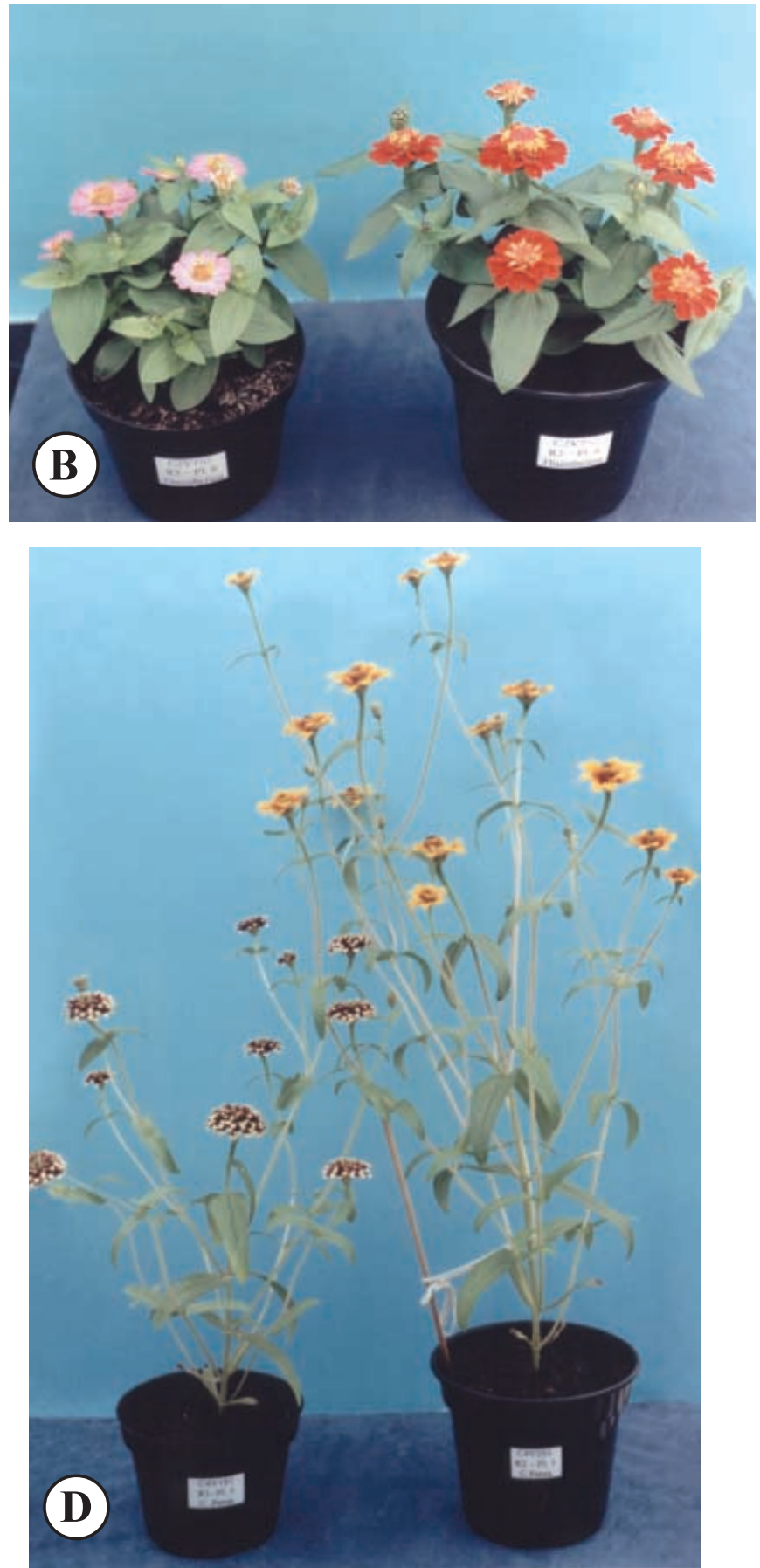

Figura 1. Plantas de 'Profusion Cherry'(A), 'Thumbelina'(B), 'Lilliput'(C) e 'Persian Carpet'(D) em floração, produzidas em vasos de 0,6 e 1,3 L, no outono, em Jaboticabal (SP) 
O comprimento de ramos laterais e a altura da parte aérea não foram influenciados pelo sistema de condução (Tabela 1), provavelmente em virtude de a gema floral apical do caule principal não mais estar exercendo dominância apical, no momento em que foi removida. Nos cultivares de zínia estudados, observou-se maior alongamento do caule principal e dos ramos laterais após o aparecimento da gema floral apical. Conforme BERNIER et al. (1985), em muitas espécies, a iniciação floral e o crescimento vegetativo são processos associados, reduzindo a iniciação floral a dominância apical, permitindo o desenvolvimento dos ramos laterais.

Na Tabela 1, verifica-se que, quando os cultivares se desenvolveram no vaso menor, apresentaram comprimento de ramos laterais e área foliar total significativamente menores comparado ao vaso maior. A redução no tamanho do vaso de cultivo pode restringir o desenvolvimento do sistema radicular e reduzir a biossíntese e a translocação de citocininas, giberelinas e aminoácidos específicos das raízes para a parte aérea, reduzindo a expansão foliar e o crescimento de ramos (CARMI \& HEUER, 1981; SPIKER et al., 1976).

Houve interação significativa entre cultivares e tamanho de vaso, para a largura da parte aérea (Tabela 1). Para 'Profusion Cherry', não se verificou diferença significativa na largura da parte aérea entre plantas produzidas nos vasos de 0,6 e 1,3 L (Tabela 3 ), indicando que a redução no tamanho do vaso não foi suficiente para influenciar a largura da parte aérea. Para os demais cultivares, as plantas cultivadas no vaso de 0,6 L apresentaram menor largura da parte aérea comparado ao vaso de 1,3 L. Nos dois tama- nhos de vasos utilizados, 'Thumbelina' apresentou a menor largura da parte aérea e 'Persian Carpet' a maior. 'Profusion Cherry' e 'Thumbelina', por apresentarem aspecto compacto (Figura 1) e porte baixo (Tabela 1), terão menor probabilidade de sofrer danos na folhagem durante o manuseio, transporte e comercialização, comparados ao 'Lilliput' e 'Persian Carpet', que apresentaram maiores valores de altura da parte aérea e comprimento de ramos laterais (Tabela 1).

'Profusion Cherry' e 'Thumbelina' apresentaram maior porcentagem de massa seca de folhas, menor porcentagem de massa seca de ramos laterais (Tabela 4) e de caule principal (Tabelas 4 e 5) comparados ao 'Persian Carpet' e 'Lilliput'. Essa característica de distribuição de massa seca proporcionou ao 'Profusion Cherry' e 'Thumbelina' aspecto mais compacto comparado aos demais cultivares.

'Profusion Cherry', 'Thumbelina' e 'Lilliput' apresentaram porcentagem de massa seca de capítulos significativamente maior em comparação ao 'Persian Carpet'. A maior porcentagem de massa seca de botões florais foi apresentada por 'Profusion Cherry', conferindo-lhe maior potencial de desenvolvimento de novos capítulos, quando as plantas deixarem a unidade produtora (Tabela 4).

'Profusion Cherry' apresentou distribuição de massa seca mais adequada à produção de plantas envasadas e 'Thumbelina', maior acúmulo de massa no caule principal e menor nos botões florais comparado ao 'Profusion Cherry', 'Lilliput' e 'Persian Carpet', pelo maior acúmulo de massa seca nos ramos laterais e caule principal em relação a outras partes, como as folhas, revelaram arquitetura inadequada para uso em vaso (Tabelas 4 e 5).

Tabela 3. Largura da parte aérea de cultivares de zínia na floração, desenvolvidos em vasos de diferentes tamanhos

\begin{tabular}{|c|c|c|c|}
\hline \multirow{3}{*}{ Cultivar } & \multicolumn{2}{|c|}{ Tamanho de vaso } & \multirow{3}{*}{ d.m.s. } \\
\hline & $0,60 \mathrm{~L}$ & $1,30 \mathrm{~L}$ & \\
\hline & \multicolumn{2}{|c|}{ Largura da parte aérea } & \\
\hline 'Profusion Cherry' & $26,13 \mathrm{aB}$ & $28,69 \mathrm{aB}$ & \\
\hline 'Thumbelina' & $20,69 \mathrm{bC}$ & $23,75 \mathrm{aC}$ & 2,75 (V d. C) \\
\hline 'Lilliput' & $24,84 \mathrm{bB}$ & $31,97 \mathrm{aB}$ & $3,64(\mathrm{C} \mathrm{d} . \mathrm{V})$ \\
\hline 'Persian Carpet' & $39,25 \mathrm{bA}$ & $49,13 \mathrm{aA}$ & \\
\hline
\end{tabular}

Médias seguidas de letras iguais, minúsculas nas linhas e maiúsculas nas colunas, não diferem entre si (Tukey 5\%); d.m.s. = diferença mínima significativa; $\mathrm{V}$ d. $\mathrm{C}=$ médias de tamanho de vaso dentro de cultivares; $\mathrm{C} \mathrm{d}$. $\mathrm{V}=$ médias de cultivares dentro de tamanho de vaso. 
Tabela 4. Porcentagem de massa seca de folhas, caule principal, ramos laterais, capítulos, botões florais e raízes, e massa seca total da planta (g) de cultivares de zínia na floração, desenvolvidos em vasos de diferentes tamanhos e sistemas de condução

\begin{tabular}{|c|c|c|c|c|c|c|c|c|}
\hline Fator & Variável & Folhas & $\begin{array}{c}\text { Caule } \\
\text { principal }\end{array}$ & $\begin{array}{l}\text { Ramos } \\
\text { laterais }\end{array}$ & $\begin{array}{l}\text { Capí- } \\
\text { tulos }\end{array}$ & $\begin{array}{l}\text { Botões } \\
\text { florais" }\end{array}$ & Raízes & Total \\
\hline & & & & & & & & $\mathrm{g}$ \\
\hline \multirow[t]{5}{*}{ Cultivar } & 'Profusion Cherry' & $24,9 \mathrm{a}$ & $4,7 \mathrm{c}$ & $22,2 \mathrm{c}$ & $32,5 \mathrm{a}$ & $2,5 \mathrm{a}$ & $8,6 \mathrm{~d}$ & $4,65 \mathrm{~d}$ \\
\hline & 'Thumbelina' & $24,2 \mathrm{a}$ & $7,9 \mathrm{~b}$ & $24,0 \mathrm{c}$ & $32,5 \mathrm{a}$ & $2,1 \mathrm{~b}$ & $8,2 \mathrm{c}$ & $6,03 \mathrm{c}$ \\
\hline & 'Lilliput' & $18,7 \mathrm{c}$ & $11,1 \mathrm{a}$ & $30,4 \mathrm{~b}$ & $30,1 \mathrm{a}$ & $1,8 \mathrm{c}$ & $8,6 \mathrm{~b}$ & $8,38 \mathrm{a}$ \\
\hline & 'Persian Carpet' & $21,0 \mathrm{~b}$ & $10,1 \mathrm{a}$ & 37,9 a & $18,2 \mathrm{~b}$ & $2,1 \mathrm{~b}$ & $9,8 \mathrm{a}$ & $7,06 \mathrm{~b}$ \\
\hline & d.m.s. & 1,5 & 1,1 & 2,3 & 3,0 & 0,1 & 1,0 & 0,99 \\
\hline \multirow[t]{3}{*}{ Tamanho de vaso } & $0,60 \mathrm{~L}$ & $21,9 \mathrm{a}$ & $9,2 \mathrm{a}$ & $27,2 \mathrm{~b}$ & $29,3 \mathrm{a}$ & $2,0 \mathrm{~b}$ & $8,1 \mathrm{~b}$ & $5,49 \mathrm{~b}$ \\
\hline & $1,30 \mathrm{~L}$ & $22,5 \mathrm{a}$ & $7,7 \mathrm{~b}$ & $30,1 \mathrm{a}$ & $27,3 \mathrm{~b}$ & $2,2 \mathrm{a}$ & $9,4 \mathrm{a}$ & $7,57 \mathrm{a}$ \\
\hline & d.m.s. & 0,8 & 0,6 & 1,2 & 1,6 & 0,1 & 0,5 & 0,53 \\
\hline \multirow{3}{*}{$\begin{array}{l}\text { Sistema de } \\
\text { condução }\end{array}$} & Livre brotação de gemas & $22,1 \mathrm{a}$ & $9,1 \mathrm{a}$ & $27,7 \mathrm{~b}$ & $29,0 \mathrm{a}$ & $2,1 \mathrm{a}$ & $8,6 \mathrm{a}$ & $6,38 \mathrm{a}$ \\
\hline & Desponte da gema floral apical & 22,4 a & $7,8 \mathrm{~b}$ & 29,6 a & 27,6 a & $2,1 \mathrm{a}$ & 9,0 a & $6,68 \mathrm{a}$ \\
\hline & d.m.s. & 0,8 & 0,6 & 1,2 & 1,6 & 0,1 & 0,5 & 0,53 \\
\hline \multicolumn{2}{|c|}{ Teste F (Cultivar - C) } & $50,85^{* *}$ & $92,18 * *$ & $137,72 * *$ & $75,94 * *$ & $79,79 * *$ & $6,90 * *$ & $36,52 * *$ \\
\hline \multicolumn{2}{|c|}{ Teste F (Tamanho de vaso - V) } & $2,11 \mathrm{~ns}$ & $26,44 * *$ & $23,87 * *$ & $6,28 *$ & $26,04 * *$ & $24,83 * *$ & $62,75^{* *}$ \\
\hline \multicolumn{2}{|c|}{ Teste F (Sistema de condução - S) $0,48 \mathrm{~ns}$} & $18,15^{* *}$ & $9,64 * *$ & $3,21 \mathrm{~ns}$ & $0,00 \mathrm{~ns}$ & $2,64 \mathrm{~ns}$ & $1,35 \mathrm{~ns}$ & \\
\hline \multicolumn{2}{|c|}{ Teste F (Interação C x V) } & $2,56 \mathrm{~ns}$ & $3,83 *$ & $0,75 \mathrm{~ns}$ & $1,23 \mathrm{~ns}$ & $1,23 \mathrm{~ns}$ & $2,42 \mathrm{~ns}$ & $3,73^{*}$ \\
\hline \multicolumn{2}{|c|}{ Teste F (Interação C x S) } & $1,10 \mathrm{~ns}$ & $2,66 \mathrm{~ns}$ & $0,80 \mathrm{~ns}$ & $0,12 \mathrm{~ns}$ & $0,72 \mathrm{~ns}$ & $3,04 *$ & $1,68 \mathrm{~ns}$ \\
\hline \multicolumn{2}{|c|}{ Teste F (Interação V x S) } & $0,41 \mathrm{~ns}$ & $2,44 \mathrm{~ns}$ & $0,56 \mathrm{~ns}$ & $1,23 \mathrm{~ns}$ & $0,03 \mathrm{~ns}$ & $3,71 \mathrm{~ns}$ & $4,65^{*}$ \\
\hline \multicolumn{2}{|c|}{ Teste F (Interação C x V x S) } & $0,26 \mathrm{~ns}$ & $0,23 \mathrm{~ns}$ & $0,97 \mathrm{~ns}$ & $0,26 \mathrm{~ns}$ & $0,68 \mathrm{~ns}$ & $0,34 \mathrm{~ns}$ & $1,07 \mathrm{~ns}$ \\
\hline \multicolumn{2}{|c|}{ Coeficiente de variação (\%) } & 7,35 & 14,04 & 8,45 & 11,04 & 5,83 & 11,92 & 16,00 \\
\hline
\end{tabular}

Médias seguidas de letras iguais nas colunas não diferem entre si (Tukey 5\%); ns não significativo a 5\% de probabilidade; * e **, respectivamente, significativo a $5 \%$ e $1 \%$ de probabilidade; d.m.s. = diferença mínima significativa; ${ }^{*}$ dados transformados em $\ln (\mathrm{x}+5,0)$ para análise e apresentação.

Tabela 5. Porcentagem de massa seca do caule principal de cultivares de zínia na floração, desenvolvidos em vasos de diferentes tamanhos

\begin{tabular}{lccc}
\hline \multirow{2}{*}{ Cultivar } & \multicolumn{2}{c}{ Tamanho de vaso } & \multirow{2}{*}{ d.m.s. } \\
\cline { 2 - 3 } & $0,60 \mathrm{~L}$ & $1,30 \mathrm{~L}$ & \\
\cline { 2 - 3 } & Porcentagem de massa seca do caule principal & $4,3 \mathrm{aC}$ & $1,20(\mathrm{~V}$ d. C) \\
\hline 'Profusion Cherry' & $5,1 \mathrm{aC}$ & $7,6 \mathrm{aB}$ & $1,60(\mathrm{C}$ d. V) \\
'Thumbelina' & $8,1 \mathrm{aB}$ & $9,6 \mathrm{bA}$ & \\
'Lilliput' & $12,6 \mathrm{aA}$ & $9,2 \mathrm{bAB}$ & \\
'Persian Carpet' & $11,0 \mathrm{aA}$ &
\end{tabular}

Médias seguidas de letras iguais, minúsculas nas linhas e maiúsculas nas colunas, não diferem entre si (Tukey 5\%); d.m.s. = diferença mínima significativa; $\mathrm{V}$ d. $\mathrm{C}=$ médias de tamanho de vaso dentro de cultivares; $\mathrm{C}$ d. $\mathrm{V}=$ médias de cultivares dentro de tamanho de vaso. 
Observou-se, em todos os cultivares, diminuição na porcentagem de massa seca de ramos laterais, de botões florais, de raízes (Tabelas 4 e 6 ) e na massa seca total (Tabelas 4 e 7), quando cultivados no vaso menor. Verificou-se, entretanto, aumento na porcentagem de massa seca de capítulo com a diminuição no tamanho do vaso de cultivo (Tabela 4).

A redução no tamanho do vaso, ao promover a redução no volume de substrato disponível ao crescimento das raízes, pode reduzir o crescimento do sistema radicular e da parte aérea. Conforme RICHARDS \& ROWE (1977), as raízes fornecem hormônios para o crescimento da parte aérea, especialmente citocininas, as quais mantêm a capacidade de dreno e crescimento da parte aérea (GEISLER \& FERREE, 1984). Ademais, KRIZEK et al. (1985) relatam que a restrição no volume de substrato disponível ao crescimento das raízes pode causar alterações fisiológicas mesmo em plantas sem estresse hídrico e nutricional, como provavelmente ocorreu no presente experimento, em que as plantas não apresentaram sintomas visuais de deficiência hídrica e nutricional.
O estresse causado pela restrição ao desenvolvimento do sistema radicular talvez tenha promovido aumento nos níveis de etileno (KENT \& YANG, 1980) e ácido abscísico (CARMI, 1995; LIU \& LATIMER, 1995), intensificando a atividade dos capítulos como drenos, o que favoreceria a produção de sementes antes de as plantas senescerem. As zínias são plantas monocárpicas, razão por que a planta toda senesce e morre após a floração.

O desponte da gema floral apical reduziu a porcentagem de massa seca do caule principal e aumentou a de ramos laterais (Tabela 4). O desponte impediu o desenvolvimento da haste floral e do capítulo apical, o que, possivelmente, impediu o acúmulo de massa no caule principal, e provavelmente, redirecionou o acúmulo de massa para os ramos laterais. O desponte prolongou o ciclo vegetativo, favorecendo o crescimento dos ramos laterais, e proporcionou maior acúmulo de massa nas raízes de 'Persian Carpet' comparado aos demais cultivares (Tabela 6).

Tabela 6. Porcentagem de massa seca de raízes, de cultivares de zínia na floração, desenvolvidos em diferentes sistemas de condução

\begin{tabular}{lccc}
\hline & \multicolumn{2}{c}{ Sistema de condução } & d.m.s. \\
\cline { 2 - 3 } Cultivar & $\begin{array}{c}\text { Livre brotação } \\
\text { de gemas }\end{array}$ & $\begin{array}{c}\text { Desponte da gema } \\
\text { floral apical }\end{array}$ & \\
\cline { 2 - 3 } & \multicolumn{2}{c}{ Porcentagem de massa seca de raízes } & \\
\hline 'Profusion Cherry' & $8,7 \mathrm{aA}$ & $8,4 \mathrm{aB}$ & 1,10 (S d. C) \\
'Thumbelina' & $8,1 \mathrm{aA}$ & $8,2 \mathrm{aB}$ & 1,40 (C d. S) \\
'Lilliput' & $8,5 \mathrm{aA}$ & $8,6 \mathrm{aB}$ & \\
'Persian Carpet' & $8,9 \mathrm{bA}$ & $10,6 \mathrm{aA}$ & \\
\hline
\end{tabular}

Médias seguidas de letras iguais, minúsculas nas linhas e maiúsculas nas colunas, não diferem entre si (Tukey 5\%); d.m.s. = diferença mínima significativa; $\mathrm{S}$ d. $\mathrm{C}$ = médias de sistema de condução dentro de cultivares; $\mathrm{C}$ d. $\mathrm{S}$ = médias de cultivares dentro de sistemas de condução.

Tabela 7. Massa seca total da planta (g), de cultivares de zínia na floração, desenvolvidos em vasos de diferentes tamanhos

\begin{tabular}{|c|c|c|c|}
\hline \multirow{3}{*}{ Cultivar } & \multicolumn{2}{|c|}{ Tamanho de vaso } & \multirow{3}{*}{ d.m.s } \\
\hline & $0,60 \mathrm{~L}$ & $1,30 \mathrm{~L}$ & \\
\hline & \multicolumn{2}{|c|}{ Massa seca total da planta } & \\
\hline & 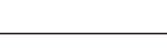 & + & \\
\hline 'Profusion Cherry' & $4,10 \mathrm{bC}$ & $5,21 \mathrm{aD}$ & \\
\hline 'Thumbelina' & $5,30 \mathrm{bBC}$ & $6,76 \mathrm{aC}$ & 1,05 (V d. C) \\
\hline 'Lilliput' & $6,70 \mathrm{bA}$ & $10,06 \mathrm{aA}$ & $1,39(\mathrm{C} \mathrm{d} . \mathrm{V})$ \\
\hline 'Persian Carpet' & $5,88 \mathrm{bAB}$ & $8,23 \mathrm{aB}$ & \\
\hline
\end{tabular}

Médias seguidas de letras iguais, minúsculas nas linhas e maiúsculas nas colunas, não diferem entre si (Tukey 5\%); d.m.s. = diferença mínima significativa; $\mathrm{V}$ d. $\mathrm{C}$ = médias de tamanho de vaso dentro de cultivares; $\mathrm{C}$ d. V = médias de cultivares dentro de tamanho de vaso. 
A maior porcentagem de cobertura do vaso pela parte aérea foi apresentada por 'Profusion Cherry', seguido em ordem decrescente por 'Thumbelina', 'Lilliput' e 'Persian Carpet' (Tabela 8). Quanto maior a porcentagem de cobertura do vaso pela parte aérea, melhor o aspecto visual da planta, indicando parte aérea bem fechada e compacta. 'Profusion Cherry' também apresentou capítulos e o índice de colheita de folhagem e capítulos significativamente maiores, comparado aos demais cultivares. Nas plantas envasadas floridas, os produtos de colheita de importância econômica são folhagem e flores. Assim, 'Profusion Cherry' mostra-se mais vantajoso, acumulando mais material nas folhas e capítulos, comparado aos demais. O tamanho de vaso e o sistema de condução não influenciaram significativamente no diâmetro de capítulos e no índice de colheita de folhagem e capítulos.
'Profusion Cherry' e 'Persian Carpet' apresentaram menor ciclo de produção, considerados precoces comparados ao 'Lilliput', que apresentou ciclo de produção mais longo e, 'Thumbelina', que ocupou posição intermediária (Tabela 8). Para todos os cultivares, o desponte da gema floral apical aumentou o ciclo de produção, visto que não permitiu o desenvolvimento do capítulo apical no caule principal, o primeiro a se abrir nas plantas dos cultivares estudados. Assim, o primeiro capítulo a se abrir passa a ser o primeiro desenvolvido em um dos ramos laterais, aumentando, desse modo, o número de dias para a abertura do primeiro capítulo e o ciclo de produção. O tamanho do vaso não influenciou significativamente o ciclo de produção. Provavelmente, a redução no tamanho do vaso não foi suficiente para influenciar o florescimento.

Tabela 8. Porcentagem de cobertura do vaso pela parte aérea, diâmetro de capítulos, índice de colheita de folhagem e capítulos (ICFC) e ciclo de produção de cultivares de zínia, quando desenvolvidos em vasos de diferentes tamanhos e sistemas de condução

\begin{tabular}{|c|c|c|c|c|c|}
\hline \multirow[t]{2}{*}{ Fator } & Variável & $\begin{array}{c}\text { Cobertura do vaso } \\
\text { pela parte aérea }\end{array}$ & $\begin{array}{c}\text { Diâmetro } \\
\text { de capítulos }\end{array}$ & ICFC & $\begin{array}{l}\text { Ciclo de } \\
\text { produção }\end{array}$ \\
\hline & & $\% *$ & $\mathrm{~cm}$ & $\mathrm{~g} / \mathrm{g}$ & dias \\
\hline \multirow[t]{5}{*}{ Cultivar } & 'Profusion Cherry' & $87,3 \mathrm{a}$ & $4,74 \mathrm{a}$ & $0,65 \mathrm{a}$ & $51,5 \mathrm{c}$ \\
\hline & 'Thumbelina' & $80,8 \mathrm{~b}$ & $3,77 \mathrm{~b}$ & $0,60 \mathrm{~b}$ & $59,2 \mathrm{~b}$ \\
\hline & 'Lilliput' & $62,2 \mathrm{c}$ & $3,60 \mathrm{bc}$ & $0,50 \mathrm{c}$ & $61,5 \mathrm{a}$ \\
\hline & 'Persian Carpet' & $30,2 \mathrm{~d}$ & $3,39 \mathrm{c}$ & $0,43 \mathrm{~d}$ & $50,1 \mathrm{c}$ \\
\hline & d.m.s. & 5,9 & 0,22 & 0,03 & 1,5 \\
\hline \multirow[t]{3}{*}{ Tamanho de vaso } & $0,60 \mathrm{~L}$ & $65,0 \mathrm{a}$ & $3,83 \mathrm{a}$ & $0,55 \mathrm{a}$ & $56,0 \mathrm{a}$ \\
\hline & $1,30 \mathrm{~L}$ & $65,3 \mathrm{a}$ & $3,93 \mathrm{a}$ & $0,54 \mathrm{a}$ & $55,2 \mathrm{a}$ \\
\hline & d.m.s. & 3,2 & 0,12 & 0,01 & 0,8 \\
\hline \multirow[t]{3}{*}{ Sistema de condução } & Livre brotação de gemas & $65,3 \mathrm{a}$ & $3,88 \mathrm{a}$ & $0,55 \mathrm{a}$ & $54,9 \mathrm{~b}$ \\
\hline & Desponte da gema floral apical & 64,9 a & $3,87 \mathrm{a}$ & $0,54 \mathrm{a}$ & $56,3 \mathrm{a}$ \\
\hline & d.m.s. & 3,2 & 0,12 & 0,01 & 0,8 \\
\hline \multicolumn{2}{|l|}{ Teste F (Cultivar - C) } & $268,61 * *$ & $109,56^{* *}$ & $227,33 * *$ & $189,96^{* *}$ \\
\hline \multicolumn{2}{|c|}{ Teste F (Tamanho de vaso - V) } & $0,03 \mathrm{~ns}$ & $2,98 \mathrm{~ns}$ & $0,05 \mathrm{~ns}$ & $3,50 \mathrm{~ns}$ \\
\hline \multicolumn{2}{|c|}{ Teste F (Sistema de condução - S) } & $0,05 \mathrm{~ns}$ & $0,01 \mathrm{~ns}$ & $2,65 \mathrm{~ns}$ & $13,16^{* *}$ \\
\hline \multicolumn{2}{|c|}{ Teste F (Interação C x V) } & $0,40 \mathrm{~ns}$ & $0,11 \mathrm{~ns}$ & $0,38 \mathrm{~ns}$ & $2,49 \mathrm{~ns}$ \\
\hline \multicolumn{2}{|c|}{ Teste F (Interação C x S) } & $0,43 \mathrm{~ns}$ & $1,13 \mathrm{~ns}$ & $0,61 \mathrm{~ns}$ & $2,07 \mathrm{~ns}$ \\
\hline \multicolumn{2}{|c|}{ Teste F (Interação V x S) } & $0,01 \mathrm{~ns}$ & $2,24 \mathrm{~ns}$ & $0,37 \mathrm{~ns}$ & $2,11 \mathrm{~ns}$ \\
\hline \multicolumn{2}{|c|}{ Teste F (Interação C x V x S) } & $1,36 \mathrm{~ns}$ & $1,68 \mathrm{~ns}$ & $0,56 \mathrm{~ns}$ & $0,44 \mathrm{~ns}$ \\
\hline \multicolumn{2}{|c|}{ Coeficiente de variação $(\%)$} & 9,60 & 5,90 & 4,92 & 2,94 \\
\hline
\end{tabular}

Médias seguidas de letras iguais nas colunas não diferem entre si (Tukey $5 \%$ ); ns: não significativo a $5 \%$ de probabilidade; ${ }^{* *}$ significativo a $1 \%$ de probabilidade; d.m.s. = diferença mínima significativa; *dados transformados em arc sen $\sqrt{\mathrm{x}+0,05}$, para análise e apresentação. 
Considerando-se que o tamanho do vaso e o sistema de condução não influenciaram significativamente alguns parâmetros importantes na avaliação da qualidade de plantas, como o diâmetro de capítulos, altura da parte aérea, cobertura do vaso pela parte aérea e o índice de colheita de folhagem e capítulos, recomenda-se a produção dos cultivares estudados, no vaso menor $(0,60 \mathrm{~L})$ e no sistema de condução com livre brotação de gemas. As plantas produzidas no vaso menor apresentaram menor comprimento de ramos laterais e as conduzidas com livre brotação de gemas, menor ciclo de produção.

A utilização do vaso menor permite reduzir o volume de substrato usado no preenchimento dos vasos (de $0,70 \mathrm{~L} /$ vaso), contribuindo para diminuir o custo de produção e de transporte. Ademais, a utilização do vaso menor permitiria maior produção de vasos por $\mathrm{m}^{2}$ de estrutura de cultivo. Com relação ao sistema de condução em livre brotação de gemas, este não requer custos adicionais com mão-de-obra nem dispêndio de tempo, para retirada de gemas.

Os cultivares 'Lilliput' e 'Persian Carpet' não se apresentaram suficientemente baixos e compactos para a produção de plantas envasadas de boa qualidade. Mas, pelo valor ornamental de seus capítulos e sementes de baixo custo, não devem ser definitivamente descartados para uso em vaso, antes da realização de estudos, com o objetivo de verificar a viabilidade da aplicação de retardadores de crescimento na obtenção de plantas adequadas para utilização em vaso.

\section{CONCLUSÕES}

1. 'Profusion Cherry' teve o melhor desempenho na produção de plantas envasadas. 'Thumbelina' mostrou maior potencial para utilização em vaso, quando comparado aos cultivares 'Lilliput' e 'Persian Carpet', os quais não se mostraram suficientemente baixos e compactos, para produção de plantas envasadas de boa qualidade.

2. A utilização do vaso menor $(0,60 \mathrm{~L})$, comparado ao maior $(1,30 \mathrm{~L})$, revelou-se mais interessante pela qualidade das plantas formadas e em relação a aspectos econômicos da produção.

3. O desponte da gema apical atrasou o ciclo de produção e não contribuiu para melhorar a qualidade das plantas formadas. A livre brotação de gemas não atrasou o ciclo de produção e apresentou-se economicamente vantajosa, não requerendo custos adicionais com mão-de-obra.

\section{REFERÊNCIAS BIBLIOGRÁFICAS}

BERNIER, G; KINET, J.M. \& SACHS, R.M. The physiology of flowering. II. Transition to reproductive growth. Boca Raton: CRC Press, 1985. 231p.

BOYLE, T.H. The genetic resources of Latin America's herbaceous ornamental crops vital to commercial floriculture. Diversity, v.7, n.1-2, p.50-51, 1991.

BOYLE, T.H.; STIMART, D.P. \& McINTOSH, M.S. Seasonal variation in vegetative and reproductive development in Zinnia elegans Jacq. Journal of the American Society for Horticultural Science, Alexandria, v.111, n.2, p.260266, 1986.

CARMI, A. Growth, water transport and transpiration in rootrestricted plants of bean, and their relation to abscisic acid accumulation. Plant Science, Burlington, v.107, p.69-76, 1995.

CARMI, A. \& HEUER, B. The role of roots in control of bean shoot growth. Annals of Botany, London, v.48, p.519-527, 1981.

DAIE, J. Carbohydrate partitioning and metabolism in crops. Horticultural Reviews, New York, v.7, p.69-108, 1985.

GEISLER, D. \& FERREE, D.C. Response of plants to root pruning. Horticultural Reviews, New York, v.6, p.155188, 1984.

KENT, J.B. \& YANG, S.F. Stress induced ethylene production in the ethylene-requiring tomato mutant Diageotropica. Plant Physiology, Rockville, v.65, p.327-331, 1980.

KRIZEK, D.T.et al. Comparative effects of soil moisture stress and restricted root zone volume, on morphogenetic and physiological responses of soybean [(Glycine max (L.) Merr.]. Journal of Experimental Botany, Lancaster, v.36, n.162, p.25-38, 1985.

LIU, A. \& LATIMER, J.G. Water relations and abscisic acid levels of watermelon as affected by rooting volume restriction. Journal of Experimental Botany, Lancaster, v.46, p.1011-1015, 1995.

OPPENHEIM, P.P. Segmentation and target marketing in a floral market. Acta Horticulturae, Wagening, n.536, p.529-536, 2000.

PINTO, A.C.R. Efeitos de tamanho de vaso, sistema de condução e reguladores vegetais no desenvolvimento e na qualidade de cultivares de zínia. Jaboticabal: Faculdade de Ciências Agrárias e Veterinárias, UNESP. 2003. 166p. Tese (Doutorado em Agronomia).

RICHARDS, D. \& ROWE, R.N. Effects of root restriction, root prunning and 6-benzylaminopurine on the growth of peach seedlings. Annals of Botany, London, v.41, p.729740, 1977.

SPIKER, S.; MASHKAS, A. \& YUNIS, M. The effect of single and repeated gibberellic acid treatment on internode number and length in dwarf peas. Physiologia Plantarum, Copenhagen, v.31, p.1-3, 1976. 